\title{
Opinião de cirurgiões dentistas sobre atividades de preceptoria na formação de estudantes de Odontologia de uma universidade brasileira
}

\author{
Pablo Erik da Silva Lopes*; Elaine Judite de Amorim Carvalho**; Fábio Barbosa de Souza***; Sílvia \\ Regina Jamelli****; Márcia Maria Dantas Cabral de Melo******
}

* Cirurgião-Dentista graduado pela Universidade Federal de Pernambuco

** Doutora em Patologia, Professora associada, Departamento de Clínica e Odontologia Preventiva, Universidade Federal de Pernambuco

*** Doutor em Dentística, Professor Adjunto, Departamento de Prótese e Cirurgia Bucofacial, Universidade Federal de Pernambuco

**** Doutora em Odontopediatria, Professora Associada, Departamento de Clínica e Odontologia Preventiva, Universidade Federal de Pernambuco

***** Doutora em Saúde Coletiva, Professora Adjunta, Departamento de Clínica e Odontologia Preventiva, Universidade Federal de Pernambuco

Recebido em 13/12/2017. Aprovado em 06/08/2018.

\begin{abstract}
RESUMO
O objetivo deste estudo foi conhecer a opinião de cirurgiões-dentistas sobre a atividade de preceptoria desempenhada por eles nos estágios curriculares 1, 2 e 3 do curso de Odontologia da Universidade Federal de Pernambuco, realizados em unidades da Atenção Básica à Saúde no município de Recife. Realizou-se estudo descritivo e de abordagem quantitativa. De um universo de 36 cirurgiões-dentistas foram incluídos aqueles com no mínimo um ano de experiência como preceptor. Para a coleta de dados utilizou-se formulário de entrevista semiestruturada, submetido à validação de face e aplicado face a face. Todos os 26 preceptores que atenderam ao critério de inclusão foram entrevistados, sendo 80,8\% mulheres; 92,3\% com vínculo de trabalho efetivo; 96,2\% pós-graduados, sendo 80,2\% deles especialistas em Saúde da Família. Apenas $15,4 \%$ recebiam incentivos para preceptoria, mas não estavam satisfeitos; $11,2 \%$ receberam formação em preceptoria; 92,3\% demandaram capacitação para exercê-la; 69,2\% atuaram nos três tipos de estágios. Prevaleceu (76,3\%) a preferência por atuar no estágio 3 (clínico) do que nos dois outros (extraclínicos). Para 65,3\% deles a estrutura física e organizacional para exercer a preceptoria é ruim, 69,2\% avaliaram como insatisfatório o apoio institucional do serviço e 57,7\% o da universidade, apesar dos esforços da instituição formadora em promover educação permanente e presencial nos serviços. Os pontos positivos mais citados foram a troca de saberes com os estudantes e docentes e a importância de integrar o estudante ao Sistema Único de Saúde. Conclui-se que embora valorizem a preceptoria, prevaleceram insatisfações de ordem trabalhista, de condições de trabalho e de formação que precisam ser enfrentadas. Descritores: Estágios. Preceptoria. Atenção Básica à Saúde. Sistema Único de Saúde.
\end{abstract}




\section{INTRODUÇÃO}

No Brasil, o ensino e a formação profissional na Odontologia se caracterizaram historicamente por um modelo tecnicista voltado para a prática assistencial privatista e liberal, com perfil de atendimento individualizado e de alto custo, distanciada da realidade social e epidemiológica da maioria da população ${ }^{1}$. Após a criação do Sistema Único de Saúde (SUS), um grande desafio é o de reformular este setor em coerência com o conceito de saúde mais abrangente adotado ${ }^{2}$.

Mais recentemente, para adequar a formação e qualificação dos profisssionais de saúde à realidade sanitária nacional, foram formuladas diferentes estratégias e políticas para promover uma formação contextualizada. Dentre elas, destacam-se as Diretrizes Curriculares Nacionais (DCN) para os cursos da Saúde, que visam fortalecer as iniciativas de integração ensinoserviço-comunidade pela sua potência de adequação do ensino às exigências das políticas de saúde públicas no enfrentamento dos problemas prioritários de saúde ${ }^{3-10}$. Nesse contexto, os estágios curriculares exercem o papel de viabilizar o percurso formativo do aluno no SUS como uma estratégia para formar profissionais com perfil mais adequado às necessidades político-sanitárias do país ${ }^{7}$. Para os cursos de graduação em Odontologia, as DCN definem que os estágios devem ser desenvolvidos de forma articulada e com complexidade crescente ao longo do curso, com carga horária mínima de $20 \%$ da carga horária total do curso9.

Para o desenvolvimento dos estágios curriculares exigidos pela DCN e pelas normas de políticas de educação voltadas à formação dos profissionais de saúde ${ }^{11}$, o preceptor do serviço tem papel fundamental por possibilitar ao aluno construir no próprio ambiente de trabalho competências para a vida profissional futura, destacando-se a função pedagógica do preceptor $^{12,13}$.
Contudo, há percepção de séria resistência docente, discente e dos profissionais para assumirem as novas funções formativas solicitadas pelas DCN e pela Política Nacional de Educação Permanente (PNEP) ${ }^{8,10,11}$. São relatados problemas referentes à formação dos trabalhadores de saúde, às condições de trabalho, de precarização do vínculo e de apoio institucional, que se constituem como impedimentos para o bom desempenho no exercício da preceptoria de alunos ${ }^{8}$. Na área da Odontologia é recente a produção de estudos com o interesse de analisar aspectos sobre as atividades de preceptoria de alunos na Atenção Básica à Saúde (ABS). São poucos os estudos cujo o interesse é conhecer a opinião dos cirurgiõesdentistas (CD) da ABS sobre as atribuições requeridas para essa função no contexto atual da integração ensino-serviço solicitada pelas DCN e o SUS, entre outros aspectos $5,6,9$.

Diante disso, o presente estudo apresenta os resultados de uma investigação que teve por objetivo conhecer a opinião de cirurgiões-dentistas da ABS do município de Recife, sobre aspectos relacionados às atividades de preceptoria desempenhadas por eles nos estágios curriculares do curso de Odontologia da Universidade Federal de Pernambuco (UFPE), na perspectiva de oferecer uma caracterização do problema e contribuir para qualificar o planejamento dessas ações de integração ensino-serviço.

\section{METODOLOGIA}

O presente estudo se define como descritivo, de abordagem quantitativa, que analisou a opinião dos CD da ABS do Recife sobre a atividade de preceptoria desempenhada por eles nos estágios curriculares supervisionados (ECS) 1, 2 e 3 do curso de Odontologia da UFPE, além de caracterizar o perfil desses profissionais.

A área de estudo foram as unidades básicas de saúde (UBS) da Secretaria de Saúde do Recife dos Distritos Sanitários (DS) IV e V, onde são 
desenvolvidos os referidos estágios. Cada tipo de estágio possui articulação com conteúdos específicos do eixo didático do Projeto Pedagógico (PP) do curso aos quais estão vinculados ${ }^{10}$. O planejamento didático-pedagógico para os ECS visa à aquisição de competências e habilidades relacionadas às atribuições da equipe de saúde e às específicas do $\mathrm{CD}$ que atua na $\mathrm{ABS}^{3}$. As orientações teóricas são centradas nos determinantes sociais da saúde e na produção de conhecimentos que abordem o processo saúdedoença nas suas dimensões coletiva, familiar e individual. A abordagem pedagógica utiliza tecnologias ativas aluno-centradas e supervisão tutorial para promover uma práxis no mundo do trabalho na ABS, onde o estudante é estimulado a atuar como ator social e construtor crítico da sua aprendizagem.

Nesse sentido, no processo de ensinoaprendizagem pretendido para os estágios são operacionalizados os conceitos de trabalho interprofissional (núcleo-campo), processo de trabalho na ABS, ambiente, território, vigilância à saúde, promoção da saúde e intersetorialidade, educação (popular) em saúde, epidemiologia (diagnóstico socioepidemiológico), projeto de intervenção e clínica ampliada. Para os EC 1 e 2 são reservados os conteúdos referentes às ações extra-clínicas do CD (ECS1: atribuições comuns da equipe de saúde e ações coletivas; ECS2: vigilância e promoção da saúde e da saúde bucal), desenvolvidos com carga horária semestral de 90 horas. Já o ECS 3, que possui carga horária semestral de 180 horas, é reservado ao fazer em clínica ampliada, onde o estudante articula os conhecimentos adquiridos nos ECS 1 e 2 e os conhecimentos teórico-práticos das disciplinas de Clínica Integral 1 e $2^{10}$. Todas essas atividades dos estágios, realizadas junto ao preceptor, são subsidiadas por um roteiro operacional que foi elaborado em coerência com as diretrizes e as atribuições profissionais esperadas para os profissionais da $\mathrm{ABS}^{3}$.

No ECS 1, três eixos norteadores guiam as atividades: 1. Estrutura e processo de trabalho na ABS - a Estratégia Saúde da Família; 2. O território socioambiental de responsabilidade sanitária da equipe de saúde; e 3. A ABS como centro de comunicação e ordenamento da Rede de Atenção à Saúde (mapeamento da rede de saúde/bucal utilizada pela unidade de estágio). No ECS 2, são eixos norteadores das ações: 1. Práticas de vigilância à saúde no cotidiano da unidade de saúde; 2 . O território da promoção e da vigilância à saúde da unidade de saúde; 3 . Prática de vigilância da saúde bucal (diagnóstico epidemiológico de cárie dentária em grupos sociais indicados pelo preceptor e formulação da intervenção; e 4. Projeto e intervenção em promoção em saúde. No ECS 3 quatro eixos suportam as atividades em clínica ampliada vivenciada pelos estudantes: 1 . A gestão do cuidado integral, com abordagem familiar (o processo de trabalho da equipe de saúde bucal nas atividades assistenciais, prática de atendimento clínico domiciliar à acamados, em escolas, em outros equipamentos comunitários, longitudinalidade do cuidado - acompanhamento dos casos clínicos); 2. A humanização na prática organização do acesso e a construção da agenda de cuidados individuais em saúde (critérios: acolhimento e classificação de risco, ciclo de vida; demanda espontânea versus programática); 3. Integralidade das ações (resolutividade e regulação assistencial por linha de cuidado); e 4. Planejamento de projeto terapêutico singular, com supervisão do preceptor e apoio do Núcleo de Apoio à Saúde da Família (NASF).

A população do estudo foi constituída pela totalidade de $\mathrm{CD}$ que atuaram como preceptores dos ECS 1, 2 e 3, entre 2013 e 2016. Foram incluídos os preceptores que possuíam no mínimo um ano de experiência nessa função. A seleção dos participantes foi realizada por meio de planilha de registro de dados dos preceptores, que foi fornecida 
pela coordenação do curso de Odontologia da UFPE. Do total de 34 preceptores, 26 foram selecionados para serem entrevistados por terem atendido ao critério de inclusão. A coleta dos dados foi realizada por um pesquisador do curso de Odontologia da UFPE no primeiro semestre de 2017, por meio da realização de entrevistas semiestruturadas. As entrevistas foram feitas individualmente (face a face) em local reservado nas UBS de trabalho de cada preceptor que foi selecionado para participar do estudo e após a assinatura do Termo de Consentimento Livre e Esclarecido. A elaboração de um formulário de entrevista (questionário) foi sustentada por revisão bibliográfica ${ }^{2,8,14-16,26}$ e pela análise do PP do curso, com foco nos componentes de ECS na ABS e em outros documentos do $\mathrm{ECS}^{10}$.

$O$ instrumento de entrevista foi organizado em três blocos. O primeiro bloco incluiu variáveis para caracterizar o perfil sociodemográfico e aspectos relacionados ao exercício profissional e na função de preceptoria, tais como tempo de exercício profissional na $\mathrm{ABS}$, tipo de vínculo, contratado ou efetivo, nível e tipo de formação de pós-graduação; assim como incentivos financeiros para exercer a função de preceptoria. $\mathrm{O}$ segundo bloco foi composto por variáveis relacionadas à estrutura e ao processo de trabalho para o desempenho da preceptoria, tendo sido coletados dados sobre condições de trabalho, opinião sobre preferências segundo o tipo de estágio, características necessárias para a função e sobre fatores limitantes e potencializadores para o bom desenvolvimento da função preceptora e dos estágios. Já o terceiro bloco foi reservado para verificar o grau de satisfação dos preceptores com a proposta pedagógica planejada para os ECS, quanto a sentir-se preparado para exercer a função de preceptor, remuneração salarial e preceptoria. Foi realizada a validação de face do instrumento para garantir o controle de qualidade dos dados. Dois especialistas avaliaram o questionário, segundo os critérios recomendados por Freire e Silva $(2006)^{26}$, para a aprovação do instrumento.

Inicialmente foi realizada uma revisão cuidadosa do instrumento pelos pesquisadores (respondendo como se fosse o informante e corrigindo o que fosse necessário); em seguida, os avaliadores externos procederam a verificação do seu conteúdo e clareza do instrumento e emitiram parecer. Com base nas observações dos revisores foram realizadas correções. Assim, o processo de revisão do questionário foi realizado objetivando a análise crítica da compreensão, ordem das questões e aceitabilidade do instrumento no seu todo pelo entrevistador, que foi treinado para conduzir a coleta dos dados junto aos preceptores ${ }^{10,16}$.

A análise dos dados foi descritiva, com a distribuição de frequências absolutas e relativas para as variáveis categóricas. Outras medidas estatísticas (média, desvio-padrão e mediana) foram calculadas para a variável idade, considerada numericamente. O programa estatístico utilizado para digitação dos dados e obtenção dos cálculos estatísticos foi o SPSS (Statistical Package for the Social Sciences) na versão 21. O projeto de pesquisa foi aprovado pelo Comitê de Ética em Pesquisas do Centro de Ciências da Saúde da UFPE, CAAE:64005617.3.0000.5208, parecer número 1.955.899/2017.

\section{RESULTADOS}

A taxa de resposta do estudo foi de $100 \%$. Todos os 26 preceptores participantes dos ECS 1, 2 e 3 que foram incluídos neste estudo responderam ao questionário de entrevista e tiveram seus formulários analisados.

A caracterização sociodemográfica e profissional demonstrou que em relação à idade a maioria dos participantes tinha entre 34 e 50 anos $(57,7 \%)$ e $42,3 \%$ tinham entre 51 e 67 anos. A

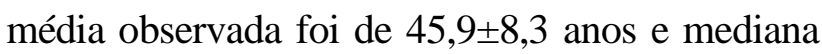
no valor de 45,5 anos. Houve predominância do sexo feminino $(80,8 \%)$ sobre o masculino $(19,2 \%)$. 
Dentre os participantes, $24(92,3 \%)$ preceptores possuem vínculo efetivo no serviço público $\mathrm{e}$ apenas dois deles $(7,7 \%)$ atuam em regime de adesão; $73 \%$ atuam na ABS do Recife há mais de sete anos e apenas 4\% trabalham há mais de 29 anos. Quanto à formação profissional, 96,2\% da amostra afirmou possuir pós-graduação, sendo a especialização o nível de formação mais frequente $(84,6 \%)$, seguido de aperfeiçoamento $(46,1 \%)$. Dentre o tipo de especialização obtida, destaca-se o fato de que $21(80,7 \%)$ dentistas possuíam o título de especialista em Saúde da Família. A maioria $(84,60 \%)$ informou não receber incentivo para o exercício da preceptoria. Dentre os $15,40 \%$ que afirmaram receber incentivo, o tipo mais frequente foi relacionado à progressão de carreira e estes afirmaram estarem insatisfeitos.

A tabela 1 contém os resultados relativos às variáveis referentes a aspectos da formação profissional em preceptoria e sobre a avaliação dessa função desempenhada pelos entrevistados. A maioria $(92,3 \%)$ considerou necessário ter formação específica para atuar como preceptor. Mais da metade da amostra $(53,8 \%)$ respondeu não ser a atividade de preceptoria uma atribuição do profissional da ABS. Sobre competências para atuar na preceptoria, predominou ter conhecimento técnico $(38,4 \%)$ seguido de gostar de ensinar $(30,7 \%)$. Sobre atividades de avaliação das ações de preceptoria $(57,7 \%)$ dos entrevistados afirmaram não ocorrer. Dentre os que afirmaram existir estes encontros $(42,3 \%)$ apontaram a UFPE (curso de Odontologia) como a instituição promotora.

$\mathrm{Na}$ tabela 2 estão apresentados os resultados sobre o processo de trabalho da preceptoria e à estruturação dos estágios. A maioria dos entrevistados $(69,20 \%)$ já atuou nos 3 tipos de ECS. Sobre o tipo de estágio de preferência, observou-se maior interesse pelo ECS $3(76,40 \%)$. A maioria $(65,3 \%)$ avaliou negativamente a estrutura organizacional da
UBS. Foram citados como problemas limitantes: o espaço físico da unidade $(23,1 \%)$, a falta de insumos $(19,2 \%)$ e a alta demanda de pacientes $(11,5 \%)$. Foi relatado pela maioria, insatisfação sobre o apoio institucional da SMS do Recife $(69,20 \%)$ e da UFPE $(57,70 \%)$. Já para as propostas de estágios $(42,30 \%)$ e supervisão dos professores $(46,10 \%)$, menos da metade demonstraram insatisfação.

Os entrevistados indicaram os pontos positivos e negativos relacionados às suas vivências como preceptor dos alunos nos ECS 1, 2 ou 3. A troca de saberes $(42,3 \%)$ foi o ponto positivo mais citado. A possibilidade de integrar o estudante ao serviço $(26,9 \%)$ e a oportunidade de se manter atualizado $(7,7 \%)$ também foram aspectos relevantes para os preceptores. Quanto aos pontos negativos, a falta de apoio e questões de estrutura foram consideradas por $26,9 \%$ deles. O despreparo dos alunos do ECS 3 (19,2\%) no desempenho clínico e a não definição de agenda (horário ou período) pela SMS do Recife $(7,7 \%)$ para se dedicarem ao acompanhamento dos estagiários também surgiram como pontos negativos.

\section{DISCUSSÃO}

Com esta investigação pode-se conhecer a opinião dos entrevistados sobre as atividades de preceptoria por eles desempenhadas junto aos estudantes dos estágios curriculares de Odontologia da UFPE. Foram evidenciados diferentes aspectos de ordem estrutural, trabalhista, formativo e de apoio interinstitucional, que estão interferindo na satisfação dos CD para realizarem a atividades de preceptoria, apesar de apresentarem satisfação com a estratégia formativa de integrar o ensino ao mundo do trabalho realizado na ABS.

A caracterização da população alvo deste estudo demonstrou que a maioria deles era do sexo feminino. Isto é coerente com outros estudos que informam haver mais CDs mulheres 
no Brasil ${ }^{19}$ e com outros realizados entre observaram o processo de feminilização da graduandos de Odontologia do país que profissão ${ }^{10,21}$.

Tabela 1. Aspectos sobre formação em preceptoria da amostra de preceptores dos Estágios Curriculares (ECS) do curso de Odontologia da UFPE na Atenção Básica à Saúde

\begin{tabular}{|c|c|c|}
\hline \multirow{2}{*}{ Variável/categoria } & \multicolumn{2}{|c|}{ Frequência } \\
\hline & n & $\%$ \\
\hline \multicolumn{3}{|l|}{ Se sente preparado para ser preceptor } \\
\hline Muito insuficiente/Insuficiente & 11 & $42,31 \%$ \\
\hline Indiferente & 2 & $7,70 \%$ \\
\hline Suficiente/ Muito suficiente & 13 & $49,90 \%$ \\
\hline \multicolumn{3}{|l|}{ Considera necessária capacitação para preceptoria } \\
\hline $\operatorname{Sim}$ & 24 & $92,30 \%$ \\
\hline Não & 2 & $7,70 \%$ \\
\hline \multicolumn{3}{|l|}{ Recebeu formação para atuar como preceptor } \\
\hline Sim & 3 & $11,50 \%$ \\
\hline Não & 22 & $84,60 \%$ \\
\hline \multicolumn{3}{|l|}{ Avaliação da formação* } \\
\hline Muito insatisfeito/Insatisfeito & - & - \\
\hline Indiferente & - & - \\
\hline Satisfeito/Muito satisfeito & 3 & $100 \%$ \\
\hline \multicolumn{3}{|l|}{ Instituição que ofereceu formação* } \\
\hline UFPE & 1 & $33,30 \%$ \\
\hline Secretaria de Saúde do Recife & 0 & - \\
\hline Outras & 2 & $66,60 \%$ \\
\hline \multicolumn{3}{|l|}{ A preceptoria é uma atribuição do profissional da ABS } \\
\hline Sim, pois o SUS é campo de prática para os estudantes & 4 & $15.30 \%$ \\
\hline Sim, pois o aluno necessita do campo de estágio para o aprendizado & 7 & $26.90 \%$ \\
\hline Não, pois não consta como obrigação trabalhista & 3 & $11.50 \%$ \\
\hline Não, deve ser uma opção individual & 6 & $23 \%$ \\
\hline Não, mas não justificaram & 5 & $19 \%$ \\
\hline \multicolumn{3}{|l|}{ Competências para ser preceptor } \\
\hline Conhecimento pedagógico & 4 & $15.3 \%$ \\
\hline Conhecimento técnico & 10 & $38.4 \%$ \\
\hline Paciência & 7 & $26.9 \%$ \\
\hline Gostar de ensinar & 8 & $30.7 \%$ \\
\hline Ter perfil para o ensino & 5 & $19 . \%$ \\
\hline Defender a formação para o SUS & 4 & $15.3 \%$ \\
\hline \multicolumn{3}{|l|}{ Realização de encontros de avaliação da preceptoria } \\
\hline Sim & 11 & $42,30 \%$ \\
\hline Não & 15 & $57,70 \%$ \\
\hline \multicolumn{3}{|l|}{ Instituição promotora da avaliação das atividades de preceptora** } \\
\hline UFPE & 9 & $81,80 \%$ \\
\hline Secretaria de Saúde do Recife & 2 & $18,20 \%$ \\
\hline TOTAL & 26 & 100 \\
\hline
\end{tabular}

*Os respondentes foram os que afirmaram (sim) na questão "Recebeu formação para ser preceptor"

** Os respondentes foram os que afirmaram ( $\operatorname{sim})$ na questão "Realização de encontros de avaliação da preceptoria" 
Tabela 2. Estrutura e processo de trabalho nas UBS para a realização dos estágios curriculares do curso de Odontologia da UFPE

\begin{tabular}{|c|c|c|}
\hline \multirow{2}{*}{ Variável/categoria } & \multicolumn{2}{|c|}{ Frequência } \\
\hline & $\mathbf{n}$ & $\%$ \\
\hline \multicolumn{3}{|c|}{ Tipo de Estágio de exercício da preceptoria } \\
\hline Apenas no estágio 2 & 1 & $3,90 \%$ \\
\hline Estágios 1 e 2 & 1 & $3,90 \%$ \\
\hline Estágios 1 e 3 & 6 & $23,00 \%$ \\
\hline Estágios 1,2 e 3 & 18 & $69,20 \%$ \\
\hline \multicolumn{3}{|c|}{ Tipo de Estágio de preferência do preceptor* } \\
\hline Estágio 1 & 5 & $29,40 \%$ \\
\hline Estágio 2 & 6 & $35,30 \%$ \\
\hline Estágio 3 & 13 & $76,40 \%$ \\
\hline \multicolumn{3}{|c|}{ Estrutura física e organizacional da UBS para a preceptoria } \\
\hline Muito insatisfeito/Insatisfeito & 17 & $65,30 \%$ \\
\hline Indiferente & - & - \\
\hline Satisfeito/Muito satisfeito & 8 & $30,70 \%$ \\
\hline \multicolumn{3}{|c|}{ Apoio institucional da SMS do Recife para a atividade de preceptoria } \\
\hline Muito insatisfeito/ Insatisfeito & 18 & $69,20 \%$ \\
\hline Indiferente & 4 & $15,40 \%$ \\
\hline Satisfeito/Muito satisfeito & 2 & $7,70 \%$ \\
\hline \multicolumn{3}{|c|}{ Apoio institucional da UFPE para a atividade de preceptoria } \\
\hline Muito insatisfeito/Insatisfeito & 15 & $57,70 \%$ \\
\hline Indiferente & 4 & $15,40 \%$ \\
\hline Satisfeito/Muito satisfeito & 8 & $23,80 \%$ \\
\hline \multicolumn{3}{|c|}{$\begin{array}{l}\text { Avaliação da supervisão dos Estágios, realizada junto ao } \\
\text { preceptor }\end{array}$} \\
\hline Muito insatisfeito/Insatisfeito & 12 & $46,10 \%$ \\
\hline Indiferente & 5 & $19,20 \%$ \\
\hline Satisfeito/Muito satisfeito & 8 & $30,80 \%$ \\
\hline \multicolumn{3}{|c|}{ Satisfação com as propostas de estágios da UFPE } \\
\hline Muito insatisfeito/Insatisfeito & 11 & $42,30 \%$ \\
\hline Indiferente & 2 & $8,0 \%$ \\
\hline Satisfeito/Muito satisfeito & 11 & $42,30 \%$ \\
\hline TOTAL & 26 & 100 \\
\hline
\end{tabular}

* Considerou-se apenas as respostas dos preceptores que atuaram nos três tipos de estágios

A maioria dos entrevistados trabalha sob o regime de vínculo efetivo, fato considerado como um fator que favorece o bom desempenho e satisfação com o trabalho ${ }^{3,8}$. Um estudo semelhante observou que preceptores com vínculo trabalhista mais permanente realizam com maior efetividade $o$ processo de trabalho solicitado para a $\mathrm{ABS}^{3}$, do que aqueles que trabalham de forma temporária, que tendem a restringir seu trabalho aos atendimentos clínicos $^{13}$. Ademais, a maioria da amostra trabalha da ABS do Recife há mais de sete anos, e tem especialidade em Saúde da Família, fato relacionado com o aumento de vagas na estratégia de saúde da família a partir de $2000^{18}$.

Esses achados podem apontar um desempenho satisfatório desses profissionais no exercício da preceptoria dos alunos do curso de Odontologia da UFPE. Para Sousa $(2014)^{14}$, o 
preceptor ao ser especialista em saúde da família ou outra área da saúde pública agrega valor tanto para a atuação na ABS como para a preceptoria. Segundo Rodrigues $(2012)^{20}$ a experiência profissional dos preceptores da ABS compartilhada com os alunos é um fator positivo registrado nas avaliações discentes sobre suas vivências nos serviços. Entretanto, discute-se, que o tempo de atuação do $\mathrm{CD}$ pode ser visto como um fator dificultador devido à formação profissional ter ocorrido sob o modelo de ensino Flexneriano ${ }^{1,10}$. Estudos evidenciam resistências na academia e nos serviços quanto à substituição do Modelo Biomédico de atuação profissional pelo requerido pelo SUS e a PNAB ${ }^{3,6,7}$.

No atual contexto da integração ensinoserviço prevista pelas DCN e demais políticas de educação em saúde ${ }^{1,4}$ é requerido ao preceptor a responsabilidade pela condução do formando no serviço $^{6}$. Contudo, neste estudo, a maioria dos entrevistados considera não ser a preceptoria uma função imposta e sim facultativa. Esses achados demonstram inconsistência dessas opiniões com as Políticas Nacionais de formação profissional em saúde ${ }^{9,11 .}$ Como também discorda de outros estudos que apontam a pertinência de formar profissionais de Odontologia na lógica de trabalho do SUS ${ }^{3,10}$, visto ser a rede pública de saúde a principal empregadora de CDs no Brasil ${ }^{15}$, o que reforça a importância da inserção precoce dos graduandos no SUS ${ }^{6}$. Ademais, tais resultados não se alinham às determinações prevista na Lei 8080/1990 da Constituição Federal ${ }^{2}$, no Capítulo II, Artigo 27, Parágrafo único, "Os serviços públicos que integram o Sistema Único de Saúde (SUS) constituem campo de prática para ensino e pesquisa, mediante normas específicas, elaboradas conjuntamente com o sistema educacional."

Sobre esses aspectos de incentivos profissionais, prevaleceu entre os entrevistados o incentivo relacionado à pontuação na progressão na carreira, já um outro estudo semelhante ${ }^{1}$ identificou a melhoria de remuneração como o incentivo prevalente. Discute-se que a função preceptora deve estar incluída no processo de trabalho dos profissionais e que um acréscimo ao salário não se faria necessário ${ }^{1}$. Essas questões necessitam serem enfrentadas com sólidas políticas de educação permanente e outras específicas voltadas a institucionalização das funções preceptoras de alunos na rede $\mathrm{SUS}^{3,11,13}$.

Discute-se que para o desempenho satisfatório da preceptoria o profissional precisaria ter conhecimento em processos educacionais para melhor lidar com as necessidades formativas do estudante $^{8}$. Metade dos entrevistados relatou se sentir preparada para atuar como preceptor, sendo que a maioria informou não ter recebido nenhuma formação. E, como observado por outros autores $^{1,10}$, a maioria considerou ser necessário obter capacitação para desenvolver as atividades de preceptoria.

Mais recentemente, são valorizadas as estratégias de educação permanente na $\mathrm{ABS}^{11}$ voltadas à resolução de problemas apresentados pela realidade, e compartilhada entre os envolvidos ${ }^{11,23,24}$. Nessa perspectiva, o curso de Odontologia da UFPE, em conjunto com a gestão de saúde bucal, realiza, periodicamente, encontros de cunho avaliativo e formativo, entre os professores supervisores e os preceptores do serviço. Para Rocha (2012) ${ }^{21}$ cabe à universidade manter os preceptores atualizados na proposta curricular e dos objetivos dos estágios, para favorecer a adesão aos objetivos e o cumprimento de pactos. Apesar disso, menos da metade dos entrevistados consideram esses encontros como um espaço onde se realiza a avaliação da preceptoria, mas houve o reconhecimento da UFPE como instituição promotora das avaliações. Contudo, essas ações promovidas na UFPE, na perspectiva da educação permanente, não foram percebidas, pela maioria dos entrevistados, supondo-se uma 
maior valorização por modelos formativos mais tradicionais $^{10,11}$.

A insatisfação dos participantes quanto à estrutura física das UBS para o desenvolvimento dos estágios foi prevalente sendo esse fato visto também em outras investigações ${ }^{1,8,10}$ nas quais foram identificadas insatisfações de preceptores da ABS relacionadas às condições físicas da unidade de saúde, à carência de insumos e sobre adequação dos horários de trabalho ao exercício dessa função, assim como foi observado, também, neste estudo.

Discute-se que embora os problemas de estrutura das UBS sejam um dificultador, estes devem servir para a reflexão crítica dos alunos na busca de soluções contextualizadas para os problemas apresentados pelo mundo real do trabalho no SUS ${ }^{1}$. Propicia-se assim, a construção de competências, habilidades e atitudes voltadas a uma atuação profissional multidimensional e de dimensões ético-políticas ${ }^{6,10}$.

A maioria dos preceptores já teve a oportunidade de atuar nos três ECS. Foi observada preferência de atuação dos CD no ECS 3, cujos objetivos estão voltados à atuação na clínica básica em saúde bucal preconizada para a $\mathrm{ABS}^{3,4}$. Essa escolha foi expressada pela possibilidade de contribuir no desenvolvimento técnico $\mathrm{e}$ profissional do estudante. Esse resultado pode estar revelando uma maior valorização pelos preceptores das atividades assistenciais de caráter individual, em detrimentos das demais atribuições de cunho coletivo e de abordagem multiprofissional exigidas para as equipes de saúde bucal ${ }^{3,23}$. Além de ser concordante com outros estudos que discutem dificuldades no processo de substituição de práticas curativas por práticas pautadas no referencial da promoção à saúde e nas diretrizes da $\mathrm{PNAB}^{21,25}$. Contudo, a inexperiência dos alunos no desempenho em atividades clínicas no ECS 3 foi um ponto negativo citado por uma parcela dos preceptores. Apesar de ser recomendada a inserção o mais precocemente possível dos alunos nos campos de estágios para adquirem as competências profissionais exigidas pela $\mathrm{DCN}^{2,9,10}$.

Mais da metade dos entrevistados demonstraram insatisfação em relação aos apoios institucionais, seja do serviço ou da universidade. A maior insatisfação foi referente ao apoio dos serviços, como a falta de programas de incentivos e indefinição de horário determinado para o desempenho dessa função, como relatado a seguir por um entrevistado: “... preceptoria é uma atividade adicional, colocada no mesmo horário das atividades cotidianas da unidade, gerando uma sobrecarga de trabalho".

Em concordância com a intencionalidade do curso Odontologia da UFPE outros autores discutem $^{18,24}$ que cabe à instituição de ensino superior preparar os preceptores que irão atuar nos seus ECS, pois nesses locais se tem a melhor estrutura e recursos humanos com qualificação para realizarem formação pedagógica. Contudo, a maior queixa dos entrevistados se referiu à inexistência de formação, apesar dos esforços dessa instituição formadora, em realizar processos compartilhados de educação permanente antes, durante e ao término dos ECS. Tais resultados apontam para a necessidade de serem instauradas rodas de conversas entre os atores envolvidos nessa integração ensino-serviço, na busca de novos consensos sobre as melhores estratégias educacionais, com vistas a responder às demandas de formação sentida pelos preceptores entrevistados e aquelas sobre condições de trabalho nos serviços.

Sousa, (2014) ${ }^{14}$ apontou a importância da tutoria do professor supervisor dos ECS ser realizada próximo ao preceptor. Apesar dos professores supervisores dos ECS realizarem suas atividades de supervisão na modalidade presencial e semipresencial de acordo com uma agenda pactuada, se observou insatisfação com tal apoio, sendo esse achado concordante com um outro 
estudo $^{15}$. Sobre esse resultado, vale considerar o relato de um entrevistado:" a falta de formação específica em preceptoria acaba gerando uma maior dependência do professor, pois os preceptores acabam não se sentindo aptos a realizarem certos aspectos da preceptoria".

Houve equilíbrio no grau de satisfação quanto às propostas dos estágios. Mas, menos da metade informou, igualmente, estar satisfeito ou muito insatisfeito, apesar da maioria ter participado dos processos de planejamento e de validação dos roteiros pedagógicos, que servem para subsidiar as atividades preceptoras junto aos alunos. Esses aspectos e dificuldades também foram observados em outros estudos ${ }^{1,10}$. Por outro lado, os entrevistados avaliaram positivamente a atividade preceptora, por integrar do aluno ao serviço e favorecer as trocas de saberes acadêmicos e profissionais, que contribuem para mantê-los atualizados. Esses achados encontraram concordâncias com outros estudos semelhantes $^{12,21}$.

Apesar de haver limitações no tipo de estudo adotado, como a baixa validade externa, se tomaram cuidados metodológicos na construção e aplicação do instrumento de coleta de dados para que os resultados expressassem de fato a opinião prestada pelos participantes minimizando o viés de informação.

Os achados deste estudo revelaram problemas que merecem esforços entre instituições para qualificar o planejamento das ações voltadas a educação permanente desses profissionais. Com a finalidade de promover uma formação inserida na realidade dos serviços de saúde do SUS, para contribuir na melhoria das condições de saúde das populações alvos das políticas de integração ensino-serviço-comunidade locais.

\section{CONCLUSÕES}

Os entrevistados demonstraram insatisfação com a política de preceptoria adotada pela instituição executora, a qual foi relacionada a questões trabalhistas, de condições de trabalho, de cunho formativo e de falta de apoio das duas instituições envolvidas. Solicitaram capacitação específica para atuação na preceptoria dos estágios e relataram problemas de ordens estruturais, operacionais e de apoio das duas instituições envolvidas, para o desenvolvimento de suas atividades preceptoras com maior satisfação. Como pontos positivos, valorizaram a troca de saberes com os alunos e professores, na perspectiva de se manterem constantemente atualizados e consideraram a importância dos estágios por integrarem o ensino dos alunos à realidade do SUS e propiciar vivencias no âmbito da ABS do Recife.

\section{ABSTRACT \\ Dentists' opinion about preceptory activity in the training of Dentistry students from a Brazilian university}

This study aimed to know the opinion of dentists on the preceptorship activities performed in the dental curricular internships 1,2 and 3 of Federal University of Pernambuco, carried out in units of basic attention to health in the city of Recife. Descriptive study and quantitative approach were carried out. From a universe of 36 dentists, were included those with at least one year of experience as preceptor. For the collection of data used A semi-structured interview, subjected to face validation and applied face to face, was used for data collection. All 26 preceptors who obeyed the inclusion criterion were interviewed, being $80.8 \%$ women; $92.3 \%$ with effective work link; $96.2 \%$ graduate, being $80.2 \%$ of them family health specialists. Only $15.4 \%$ received incentives for preceptorship, but they were not satisfied; $11.2 \%$ received training in preceptorship; $92.3 \%$ were trained to exercise it; $69.2 \%$ acted in the three types of internships. It prevailed (76.3\%) the preference for acting in Stage 3 (clinical) than in the two others (nonclinical). According to $65.3 \%$ of the interviewed, physical and organizational structure to exercise the preceptorship is bad, $69.2 \%$ evaluated the institutional support of the service as unsatisfactory and $57.7 \%$ of the university, despite 
the efforts of training institution in promoting permanent education and presence in services. The most cited positive points were the exchange of knowing with the students and faculty, and the importance of integrating the student into the unique health system. It is concluded that although they value the governess, they have prevailed dissatisfactions of labor order, working conditions and training that need to be addressed.

Descriptors: Internships. Preceptorship. Primary Health Care. Unified Health System.

\section{REFERÊNCIAS}

1. Rocha PF, Toassi RFC, Warmling CM. O ensino nos serviços de atenção primária do Sistema Único de Saúde (SUS) na formação de profissionais de saúde no Brasil. Interface (Botucatu). 2013;17(45):385-92.

2. Brasil. Ministério da Saúde. Ministério da Educação. A aderência dos cursos de graduação em enfermagem, medicina e odontologia às diretrizes curriculares nacionais - 162 p.: il. - (Série F. Comunicação e Educação em Saúde). Brasília: Ministério da Saúde; 2006.

3. Brasil. Ministério da Saúde. Secretaria de Atenção à Saúde. Política Nacional de Atenção Básica - 110 p.: il. - (Série E. Legislação em Saúde). Brasília: Ministério da Saúde; 2012.

4. Fonseca EP. As Diretrizes Curriculares Nacionais e a formação do cirurgião-dentista brasileiro. J Manag Prim Health Care. 2012; 3(2):158-78.

5. Toassi RFC, Davoglio RS, Lemos VMA. Integração ensino-serviço-comunidade: o estágio na atenção básica da graduação em Odontologia. Educ Rev. 2012; 28(4): 22342.

6. Ceccim RB, Feuerwerker LCM. O quadrilátero da formação para a área da saúde: ensino, gestão, atenção e controle social. Physis. 2004;14(1):41-65.
7. Werneck MAF, Senna MIB, Drumond MM, Lucas SD. Nem tudo é estágio: contribuições para o debate. Ciênc Saúde Colet. 2010; 15(1):221-31.

8. Trajman A, Assunção N, Venturi M, Tobias D, Toschi W, Brant V. A preceptoria na rede básica da Secretaria Municipal de Saúde do Rio de Janeiro: opinião dos profissionais de Saúde. Rev Bras Educ Méd. 2009; 33(1):2432.

9. Brasil. CNE/CES 3/2002. Diário Oficial da União, Seção 1, p. 4; Brasília, 10 de março de 2002. [Acesso em 2 jun. 2017]. Disponível em: http://portal.mec.gov.br /sesu/arquivos/pdf/0302Odontologia.pdf.

10. Melo MMDC, Souza FB, Pires IBF, Cardoso LHG. Formação em odontologia voltada para o SUS: uma avaliação discente. Rev EDUCA. 2016; 3(6):92-116.

11. Carotta F, Kawamura D, Salazar J. Educação permanente em saúde: uma estratégia de gestão para pensar, refletir e construir práticas educativas e processos de trabalhos. Saúde Soc. 2009; 18 (1):48-51.

12. Barreto VHL, Monteiro ROS, Magalhães GSG, Almeida RCC, Souza LN. Papel do preceptor da atenção primária em saúde na formação da graduação e pós-graduação da Universidade Federal de Pernambuco-um Termo de Referência. Rev Bras Educ Méd. 2011; 35(4):578-83.

13. Botti SHO, Rego S. Preceptor, supervisor, tutor e mentor: quais são seus papéis? Rev Bras Educ Méd. 2008; 32(3):363-73.

14. Sousa DP. Preceptoria em saúde bucal na atenção básica no município de Goiânia sob a perspectiva do preceptor. [Dissertação]. Goiânia: Universidade Federal de Goiás (UFG). 2013. [Acesso em 2 jun. 2017]. Disponível em: https://repositorio.bc.ufg.br/ tede/handle/tede/3842.

15. Rocha PF, Warmling CM, Toassi RFC. 
Preceptoria como modalidade de ensino na saúde: atuação e características do preceptor cirurgião-dentista da atenção primária. Saberes Plurais. 2016;1(1):96-112.

16. Boynton PM, Greenhalgh T. Selecting, designing, and developing your questionnaire. BMJ. 2004; 328 (74):131215 .

17. da Luz GW, Toassi RFC. Percepções sobre o preceptor cirurgião-dentista da Atenção Primária à Saúde no ensino da Odontologia. Rev ABENO. 2016;16(1):212.

18. Ribeiro MB. Formação pedagógica de preceptores do ensino em saúde. 1ed. Juiz de Fora: Ed. UFJF; 2011.126 p.

19. Morita MC, Haddad AE, Araújo ME. Perfil atual e tendências do cirurgião-dentista brasileiro. Dental Press. 2010;196. 98p.

20. Rodrigues CDS. Competências para a preceptoria: construção no programa de educação pelo trabalho para a saúde. [Dissertação]. Porto Alegre: Universidade Federal do Rio Grande do Sul (UFRGS). 2012. [Acesso em 15 maio 2017]. Disponível em: http://hdl.handle.net/ $\underline{10183 / 56085 .}$.

21. Rocha HC, Ribeiro VB. Curso de formação pedagógica para preceptores do internato médico. Rev Bras Educ Méd. 2012; 36(3):343-50.
22. Chaves SCL, Almeida AMFL, Rossi TRA, Santana SF, Barros SG, Santos CML. Política de Saúde Bucal no Brasil 20032014: cenário, propostas, ações e resultados. Ciênc Saúde Colet. 2017;22(6):1791-803.

23. Nickel DA, Lima FG, Silva BB. Modelos assistenciais em saúde bucal no Brasil. Cad Saúde Pública. 2008; 24(2):241-6.

24. Brasil. Ministério da Saúde. Grupo Hospitalar Conceição Residências em Saúde: Fazeres \& Saberes na Formação em Saúde. Porto Alegre, 2010. p. 191-211.

25. Feuerwerker L, Almeida M. Diretrizes curriculares e projetos pedagógicos: é tempo de ação! Rev Bras Enferm. 2003;56(4):3512.

26. Freire MCM, Silva AS. Instrumentos de coleta de dados em epidemiologia da saúde bucal. In: Antunes JLF, Peres MA. Epidemiologia da saúde bucal. 1ed. Rio de Janeiro: Guanabara Koogan; 2006. p. 37683.

\section{Correspondência para:}

Pablo Erik da Silva Lopes

e-mail: pabloerik@bol.com.br

Rua Bernardo Vieira, 14 Mangueira

55602-490 Vitória de Santo Antão/PB 